\title{
PARTICIPACIÓN DE LA UNIVERSIDAD POLITÉCNICA SALESIANA EN LA EXPLORACIÓN FLORÍSTICA DE LA RESERVA ECOLÓGICA LOS ILINIZAS
}

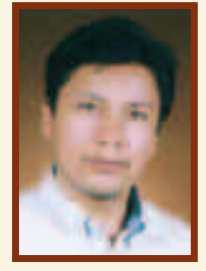

Artículo compilado por el biólogo Marco Cerna. Información extraída del Informe Final del Proyecto de Investigación "Exploración Florística de la Reserva Ecológica Los Ilinizas". Desarrollado por Philip Silvertone-Sopkin. Fotografías Marco Cerna.

\section{Antecedentes}

Este proyecto se desarrolló en la provincia de Cotopaxi con la participación de un grupo de investigadores de la Universidad del VaIle de Cali-Colombia, liderado por Philip Silverstone-Sopkin; como investigador asociado estuvo Jorge E. Ramos-Pérez (Universidad del Valle) y David A. Neill (Jardín Botánico de Missouri). En la fase de exploración participaron Marco Cerna (Universidad Politécnica Salesiana); Fabián Ibá- ñez, Jehová Figueroa, Geovanny Ibáñez, José Luis Medina, Telmo Ibáñez IAlumnos de la Carrera de Ingeniería Agropecuaria Campus Guasaganda de la Facultad de Ciencias Agropecuarias y Ambientales de la Universidad Politécnica Salesiana). El apoyo logístico estuvo a cargo del personal del Herbario Nacional del Ecuador.

El financiamiento del proyecto se lo realizó con fondos de la National Geographic Society y con la autorización de investigación científica $\mathrm{n}^{\circ}$ 008-ic-flo-dbap/ma del Ministerio del Ambiente del Ecuador.

La fase de campo de esta investigación se desarrolló desde el 2 de julio hasta el 25 de agosto de 2003 y lo resultados se pudieron presentar en julio de 2004.

\section{Introducción}

La Reserva Ecológica Los llinizas (cuyo nombre viene de Los Ilinizas, picos gemelos volcánicos) se ubica en la vertiente occidental de la Cordillera Occidental de los Andes, en el Ecuador. La mayoría de la reserva se encuentra en la provincia de Cotopaxi, pero una parte pequeña está en la provincia de Pichincha. La reserva incluye la Laguna Quilotoa, un lago

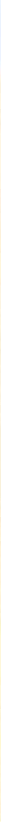




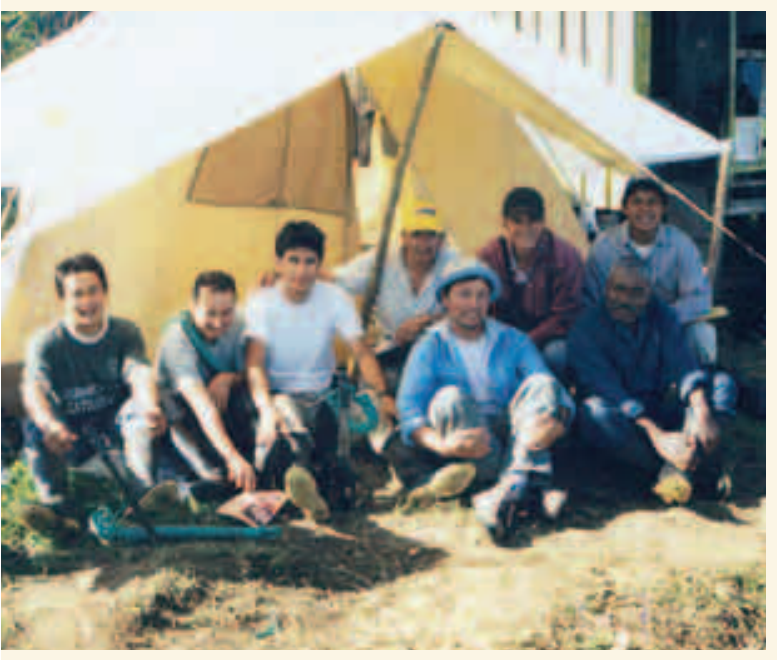

dentro de un cráter volcánico; esta zona queda por fuera de los bordes principales de la reserva. La altura sobre el nivel del mar de la reserva varía desde $800 \mathrm{~m}$ hasta $5.248 \mathrm{~m}$, y la precipitación promedio anual varía desde 1.000 $\mathrm{mm}$ hasta $2.000 \mathrm{~mm}$ (Romero-Torres, 1997).

La reserva fue fundada el 19 de diciembre de 1996, y está manejada por el Ministerio del Ambiente. El área de la reserva es 149.900 ha, de las cuales 119.100 ha originalmente eran bosque nublado y 30.800 ha pertenecían al páramo (Romero-Torres, 1997). Según el sistema de clasificación de Harling (1979), que se muestra en los mapas de vegetación en Jørgensen \& León-Yánez (1999), las zonas de vegetación en la reserva incluyen Bosque pluvial montano bajo, Bosque nublado, Pastizales y vegetación de quebradas del norte del Ecuador, Páramo de pajonal, Páramo arbustivo y de almohadillas, y Páramo desértico. Estos tipos de vegetación fueron descritos por Neill (1999). Los asentamientos humanos existieron en esta zona bien antes del establecimiento de la reserva.

\section{Objetivos}

1) Determinar las especies de plantas vasculares que se encuentran presentes en la reserva,

2) Localizar especies nuevas, endémicas y escasas.

Esta información justificaría un mayor nivel de protección para la reserva y proveería una base de datos para proyectos futuros de reforestación.

\section{Métodos}

En julio de 2002 visitaron la Reserva Ecológica Los Ilinizas Philip Silvertone-Sopkin y Marco Cerna, con el propósito de establecer las áreas a investigar, contactar a los guías, conseguir los permisos y establecer costos.

La expedición fue realizada desde el 2 de julio hasta el 25 de agosto de 2003. Los colectores fueron organizados en dos grupos. Cada grupo colectó en zonas diferentes de la reserva (un total de siete zonas).

Las colecciones fueron realizadas en bosques nublados entre 1.200 m y 3.700 m. Las zonas de colección fueron las siguientes:

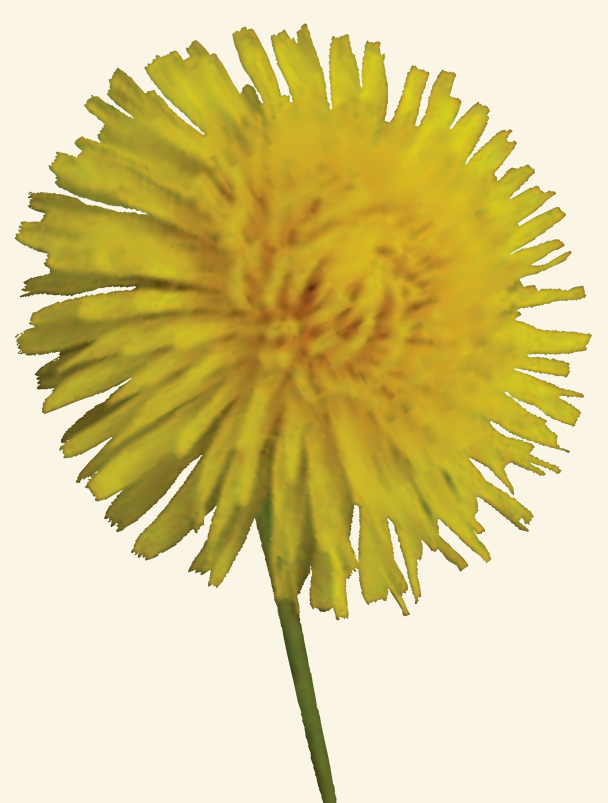

Primer Grupo (liderado por Philip A. Silverstone-Sopkin): Marco Cerna, Telmo Ibáñez Fabián Ibáñez, Jehová Figueroa, Geovanny Ibáñez, José Luis Medina.

1) Cantón La Maná: sectores El Oriente, Brasil, Lunave, Guadual (acceso desde La Carmela);

2) Cantón La Maná: vertiente norte del Cerro Tilipulo Grande (acceso desde Pucayacu y Capillas);

3) Cantón Pujili: sector sur (Sector II) de la reserva, sector Chuspitambo (acceso desde Choasillí);

4) Cantón Pujilí: sector Tilipulo (vertiente sur de la Cordillera Tilinche) (acceso desde La Esperanza $=$ El Tingo) .

Segundo Grupo (liderado por Jorge E. Ramos-Pérez): Luis Rámos y Jorge Contreras.

5) Cantón Sigchos: Cerro El Campanario, Cerro Azul, sector Zarapullo (acceso desde Las Parcelas del Alisal);

6) Cantón Sigchos: carretera Sigchos-Las Pampas, sectores Triunfo Chico y Triunfo Grande (acceso desde Sigchos);

7) Cantón Mejía (provincia de Pichincha): vertiente noroeste del Cerro Corazón (acceso desde Alóag). No fue posible colectar en la Cordillera de Almas Santas, debido a que la comunidad local no otorgó el permiso correspondiente.

Los especimenes de cada número fueron distribuidos para su identificación en:

El Herbario Nacional del Ecuador (OCNE); 
El Jardín Botánico de Missouri (MO);

Una muestra para los especialistas en cada familia en diferentes herbarios de otros países;

El Herbario de la Universidad del Valle, Cali, Colombia (CUVC);
Si nuestra estimación del número de morfoespecies está correcta, Anthurium en Los llinizas tiene un mayor número de especies que otros lugares similares en el Ecuador.

Veintiuna de las especies identificadas son endémicas del Ecuador. Una de éstas es la quinta colec-

Porcentaje de tipos de Plantas Vasculares en La Reserva Ecológica los Ilinizas

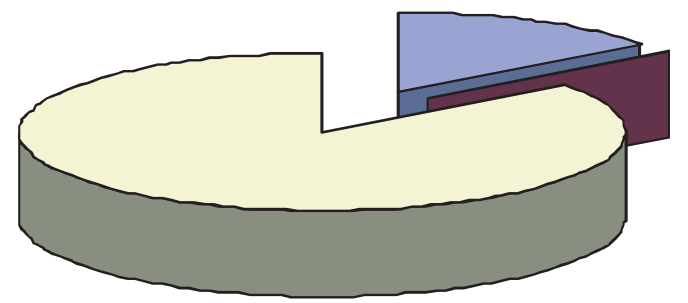

$\square$ Helechos y aliados,

$\square$ Gimnospermas

$\square$ Angiospermas

\section{RESULTADOS}

Las colecciones realizadas por los dos grupos de campo fueron en total 2.969 números.

Aproximadamente 13.000 especímenes y alrededor de 1.635 morfoespecies de plantas vasculares, de las cuales $14,7 \%$ fueron helechos y aliados; 0,1\% gimnospermas; y $85,2 \%$ angiospermas.

La mayoría de estos números todavía no han sido identificados en su especie. Sin embargo, los especimenes fueron separados en morfoespecies; así, tenemos una aproximación razonable del número de especies por familia.

La familia más diversa de angiospermas es Orchidaceae, seguida de cerca por Asteraceae. Todas las diez familias con más especies son elementos típicos de los bosques nublados menos Asteraceae y Fabaceae. ción del bejuco endémico escaso de la familia Onagraceae, Fuchsia pilaloensis Berry.

Nuestra colección de la bromeliácea Racinaea inconspicua (André) M.A. Spencer \& L.B. Sm. es el primer registro para la provincia de Cotopaxi y el tercer registro de esta especie en la colección del Her- bario Nacional del Ecuador OCNE (José M. Manzanares, com. pers.).

Seis de las especies colectadas son nuevos registros para la provincia de Cotopaxi, y una de ellas es un nuevo registro para Ecuador occidental.

Tres especies nuevas de angiospermas han sido identificadas hasta el momento; dos de ellas son Cissus (Vitaceae) y una es un Croton (Euphorbiaceae). Probablemente se encontrarán una mayor cantidad de especies nuevas en las colecciones de las familias grandes, las cuales todavía no han sido identificadas. También hay varias especies nuevas de helechos.

\section{RECOMENDACIONES}

Según expresiones de los miembros de los dos grupos de campo, éstos han observado una extensa y gran destrucción del hábitat visitado, la que ha ocurrido y todavía sigue ocurriendo en la reserva. La causa principal de esta destrucción es la creación de potreros para la cría de ganado (además de la tala de los árboles para vender su madera).

El mejor bosque remanente que encontramos fue en el sector de Brasil, accesible por mula desde La

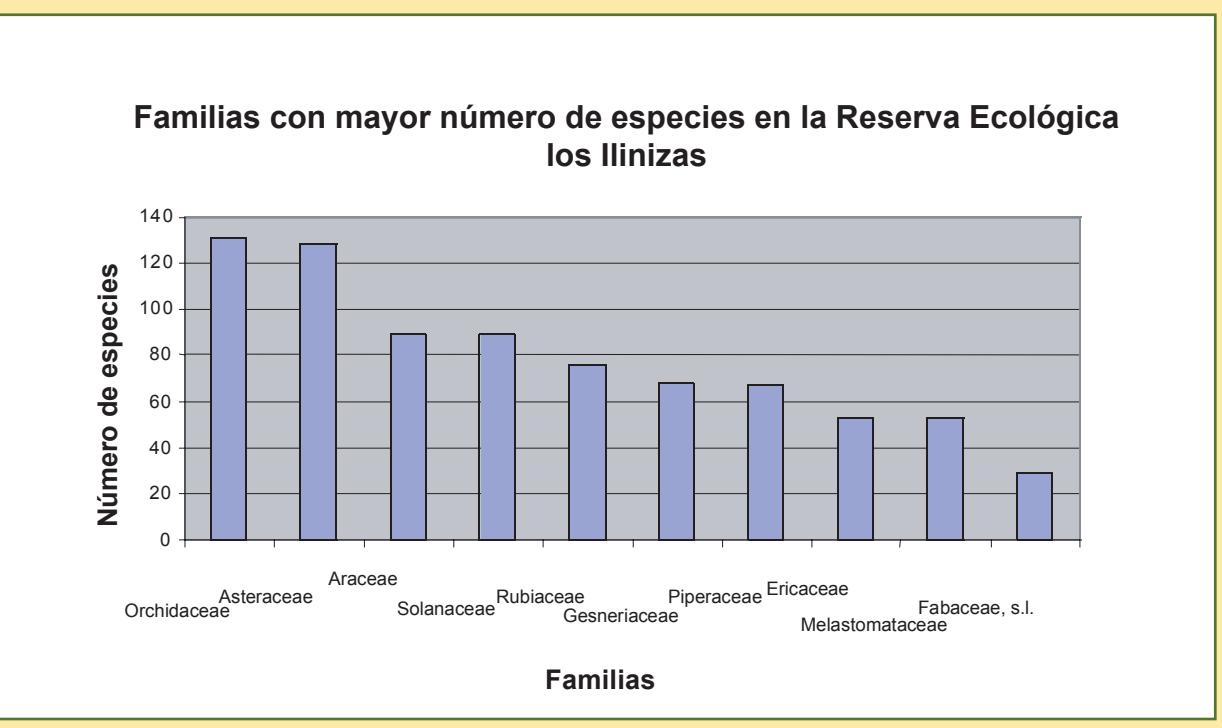


Carmela, en la vertiente occidental de la reserva, a $0^{\circ} 40^{\prime} 37^{\prime \prime} \mathrm{s}$, $79^{\circ} 05^{\prime} 09^{\prime \prime} \mathrm{W}$, a una altura de $1.480 \mathrm{~m}$. Este es un bosque primario de 700 ha, muy diverso, con muchos árboles grandes que forman un dosel a unos $30 \mathrm{~m}$. Hay muchas palmas del género Wettinia. El bosque no está invadido por bambúes del género Chusquea (suros). La fauna es diversa e incluye zainos (Tayassu).

El bosque pertenece a un solo dueño. Los campesinos locales desean comprar este bosque, dividirlo en 40 lotes, y talarlo a ras para sembrar potreros para el ganado. Se considera que este bosque merece la protección de todos, y solicitamos al Ministerio del Ambiente o una fundación particular lo adquiera para protegerlo y preservarlo.

A menos de que el nivel de protección (esencialmente cero) aumente sustancialmente; la mayoría de los bosques que permanecen en la reserva (con excepción de los que están sobre faldas muy pendientes) serán destruidos dentro de diez años.

La agricultura no es una alternativa viable versus la cría de ganado, a causa de los precios bajos de los productos agrícolas. Tal vez la mejor alternativa económica sería la cría de trucha en estanques artificiales de agua, pero esto requeriría la financiación por agencias gubernamentales o de algunas ONG.
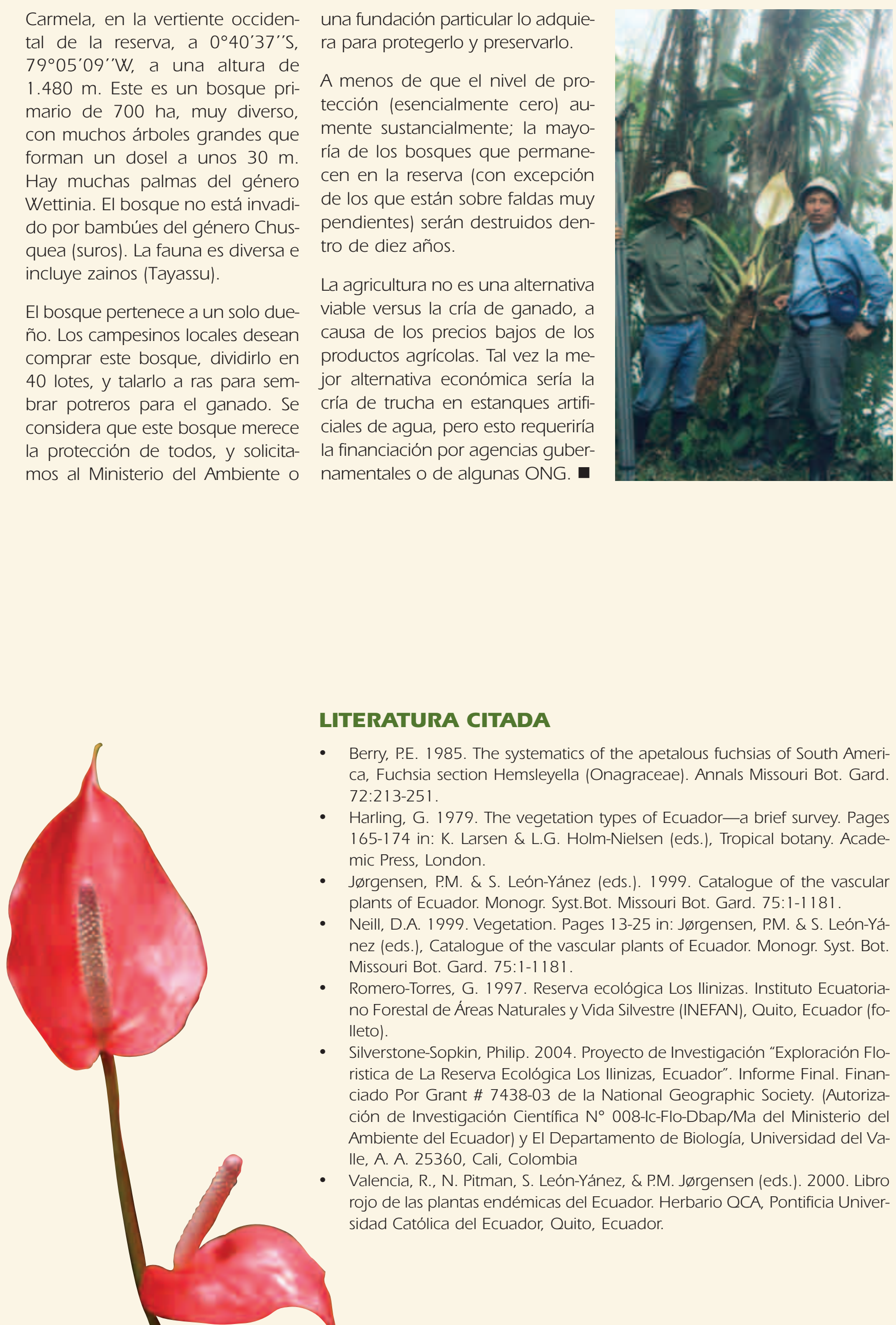

\section{LITERATURA CITADA}

- Berry, P.E. 1985. The systematics of the apetalous fuchsias of South America, Fuchsia section Hemsleyella (Onagraceae). Annals Missouri Bot. Gard. 72:213-251.

- Harling, G. 1979. The vegetation types of Ecuador-a brief survey. Pages 165-174 in: K. Larsen \& L.G. Holm-Nielsen (eds.), Tropical botany. Academic Press, London.

- Jørgensen, P.M. \& S. León-Yánez (eds.). 1999. Catalogue of the vascular plants of Ecuador. Monogr. Syst.Bot. Missouri Bot. Gard. 75:1-1181.

- Neill, D.A. 1999. Vegetation. Pages 13-25 in: Jørgensen, P.M. \& S. León-Yánez (eds.), Catalogue of the vascular plants of Ecuador. Monogr. Syst. Bot. Missouri Bot. Gard. 75:1-1181.

- Romero-Torres, G. 1997. Reserva ecológica Los Ilinizas. Instituto Ecuatoriano Forestal de Áreas Naturales y Vida Silvestre (INEFAN), Quito, Ecuador (folleto).

- Silverstone-Sopkin, Philip. 2004. Proyecto de Investigación “Exploración Floristica de La Reserva Ecológica Los Ilinizas, Ecuador". Informe Final. Financiado Por Grant \# 7438-03 de la National Geographic Society. (Autorización de Investigación Científica Nº08-Ic-Flo-Dbap/Ma del Ministerio del Ambiente del Ecuador) y El Departamento de Biología, Universidad del VaIle, A. A. 25360, Cali, Colombia

- Valencia, R., N. Pitman, S. León-Yánez, \& P.M. Jørgensen (eds.). 2000. Libro rojo de las plantas endémicas del Ecuador. Herbario QCA, Pontificia Universidad Católica del Ecuador, Quito, Ecuador. 


\section{UPS GANÓ MEJOR STAND DE FERIA AGROPECUARIA Y GANADERA EN CUENCA}

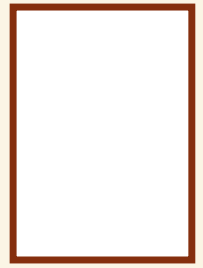

La Universidad Politécnica Salesiana se hizo acreedora al primer puesto en la elección del mejor stand de la Feria Agropecuaria y ganadera 2006 que organizó el Centro Agrícola Cantonal en el Recinto Ferial del CADECEM, durante las fiestas de la ciudad de Cuenca.

La Carrera de Ingeniería Agropecuaria presentó en esta exposición una variedad de productos elaborados por sus estudiantes en los distintos laboratorios con los que cuenta la entidad salesiana en el Campus Juan Lunardi del cantón Paute.

Entre los productos que fueron exhibidos y estuvieron a la venta, podemos destacar los elaborados lácteos (queso, yogurt, manjar), los productos frutales (conservas y mermeladas) y los elaborados cárnicos (embutidos, carnes y otros). También fueron puestas a la venta plantas ornamentales, medicinales, deshidratadas (como las que se utilizan para las aguas aromáticas).
El Vicerrector de la Universidad, sede Cuenca, Ec. Luis Tobar, dijo que la entidad se encuentra satisfecha por la acogida que tuvieron sus productos. Por su parte, el director de la Carrera, Patricio Garnica, indicó que los estudiantes fueron los responsables directos de la participación en esta feria agropecuaria. Agradeció también al público cuencano por su presencia en el stand de la UPS durante la feria.

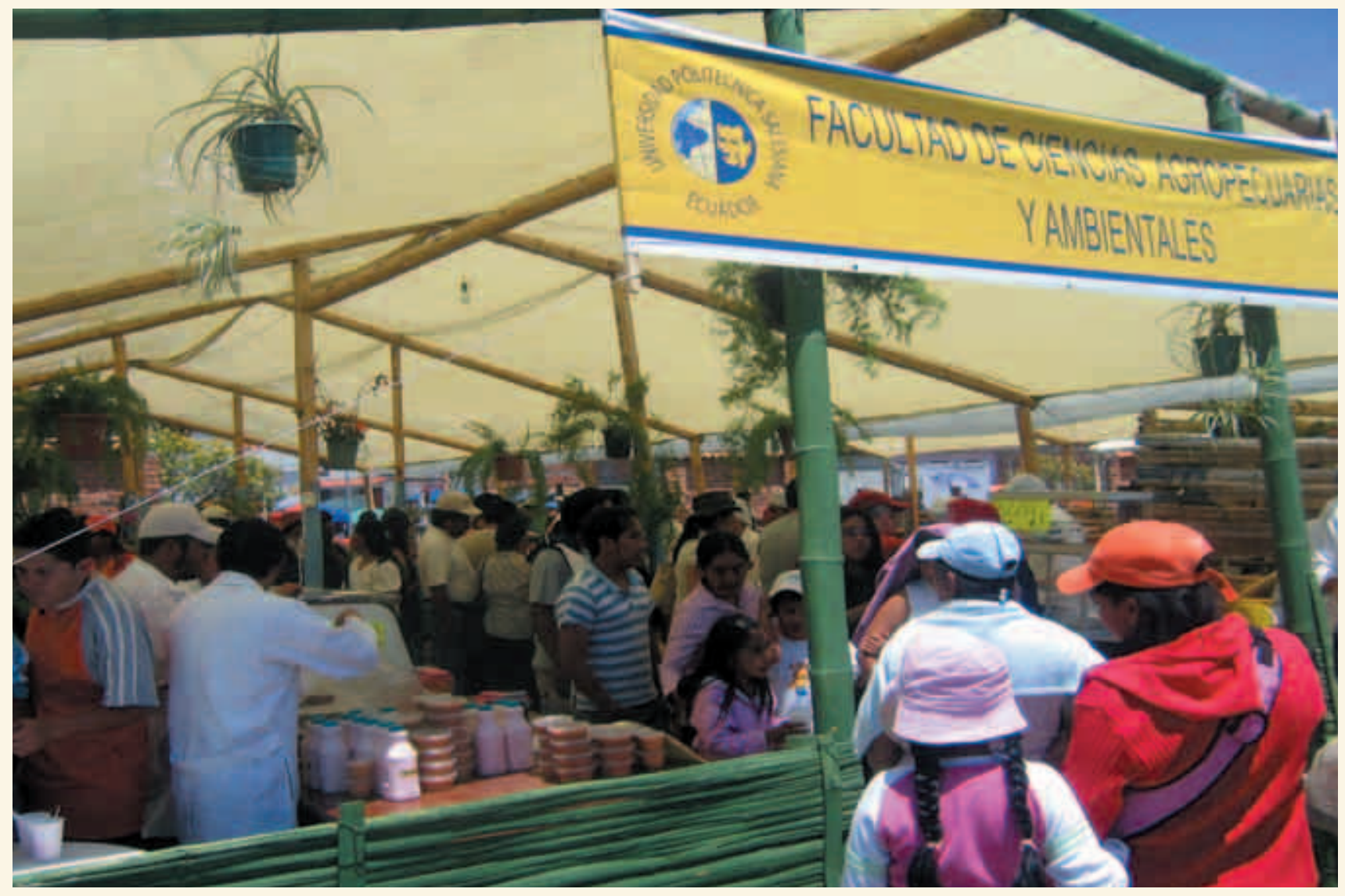

